
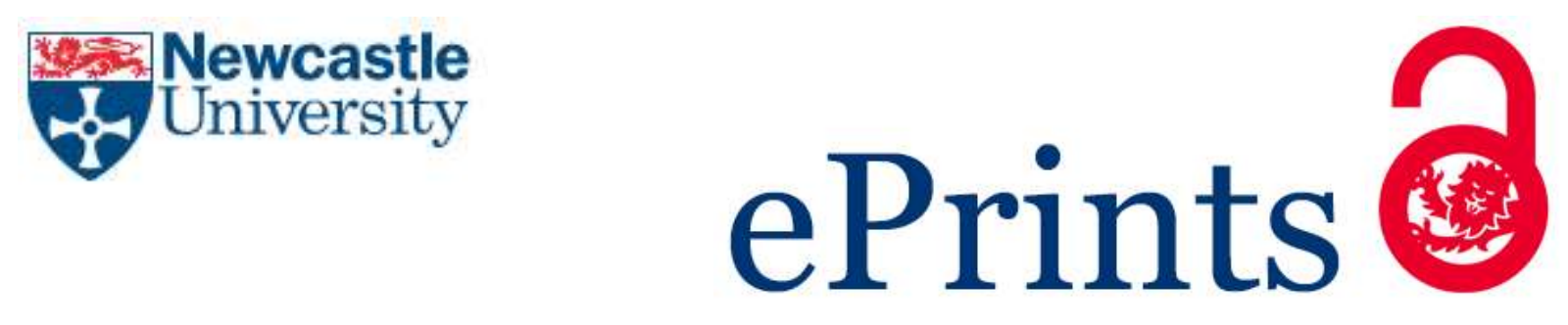

Richardson SJ, Davis DHJ, Bellelli G, Hasemann W, Meagher D, Kreisel SH, MacLullich AMJ, Cerejeira J, Morandi A. Detecting delirium superimposed on dementia: diagnostic accuracy of a simple combined arousal and attention testing procedure. International Psychogeriatrics 2017

\title{
Copyright:
}

(c) 2017 Cambridge University Press. This is the author's version of a work accepted for publication by Cambridge University Press.

DOI link to article:

https://doi.org/10.1017/S1041610217000916

Date deposited:

09/08/2017

Embargo release date:

30 November 2017

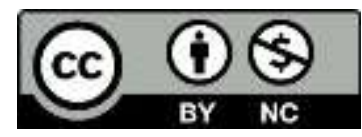

This work is licensed under a Creative Commons Attribution-NonCommercial 3.0 Unported License 


\section{Detecting delirium superimposed on dementia: diagnostic accuracy of a simple combined arousal and attention testing procedure}

Dr Sarah J Richardson*, Institute of Neuroscience, $3^{\text {rd }}$ floor Biomedical Research Building, Campus for Ageing and Vitality, Newcastle University, Newcastle upon Tyne, NE4 5PL, UK. Sarah.Richardson2@newcastle.ac.uk (+44) 01912081314

Dr Daniel H J Davis, MRC Unit for Lifelong Health and Ageing at UCL, London, UK

Dr Giuseppe Bellelli, School of Medicine and Surgery, University of Milano-Bicocca, Milan, Italy

Dr Wolfgang Hasemann, Universitätsspital Basel, Basel, Switzerland

Professor David Meagher, Graduate Entry Medical School, University of Limerick, Limerick, Ireland

Dr Stefan H Kreisel, Evangelisches Krankenhaus Bielefeld, Department of Psychiatry and Psychotherapy Bethel, Division of Geriatric Psychiatry, Bielefeld, Germany

Professor Alasdair M J MacLullich, Edinburgh Delirium Research Group, Geriatric Medicine, University of Edinburgh, Edinburgh, UK

Professor Joaquim Cerejeira, Department of Psychiatry, Hospitais da Universidade de Coimbra, Coimbra, Portugal

Dr Alessandro Morandi, Ancelle Hospital, Cremona, Italy; Geriatrics Research Group, Brescia, Italy

Corresponding author: * 


\section{ABSTRACT}

\section{Introduction}

Detecting delirium superimposed on dementia (DSD) can be challenging because assessment partly relies on cognitive tests that may be abnormal in both conditions. We hypothesised that a combined arousal and attention testing procedure would accurately detect DSD.

\section{Methods}

Patients aged $\geq 70$ years were recruited from five hospitals across Europe. Delirium was diagnosed by physicians using DSM-5 criteria using information from nurses, carers, and medical records. Dementia was ascertained by the Informant Questionnaire on Cognitive Decline in the Elderly. Arousal was measured using the Observational Scale of Level of Arousal (OSLA), which assesses eye opening, eye contact, posture, movement, and communication. Attention was measured by participants signalling each time an " $A$ " was heard when "S-A-V-E-A-H-A-A-R-T" was read out.

\section{Results}

The sample included 114 persons (mean age 82y (SD 7); 54\% women). Dementia alone was present in $25 \%(n=28)$, delirium alone in $18 \%(n=21), D S D$ in $27 \%(n=31)$, and neither in $30 \%$ $(n=34)$. Arousal and attention was assessed in $n=109$ (96\%). Using OSLA, 83\% participants were correctly identified as having delirium (sensitivity $85 \%$, specificity $82 \%$, AUROC 0.92). The attention task correctly classified $76 \%$ of participants with delirium (sensitivity $90 \%$, specificity $64 \%$, AUROC 0.80 ). Combining scores correctly classified $91 \%$ of participants with delirium (sensitivity $84 \%$, specificity $92 \%$, AUROC 0.94). Diagnostic accuracy remained high in the subgroup with dementia (93\% correctly classified, sensitivity $94 \%$, specificity $92 \%$, AUROC 0.98).

\section{Conclusions}

This combined arousal-attention assessment to detect DSD was brief yet had high diagnostic accuracy. Such an approach could have clinical utility for diagnosing DSD. 


\section{INTRODUCTION}

Delirium is an acute neuropsychiatric disorder characterised by fluctuating inattention, other cognitive deficits, altered arousal, and psychosis. It affects more than one in five hospital inpatients (Bellelli et al., 2016). When delirium occurs in someone with dementia, it is referred to as delirium superimposed on dementia (DSD). Dementia is a major risk factor for delirium, and thus many patients with delirium also have comorbid dementia, with figures ranging from $22 \%$ to $89 \%$ depending on the setting and population (Fick et al., 2002).

When compared to delirium alone, DSD is associated with worse outcomes including increased walking dependence, institutionalisation and mortality (Morandi et al., 2014) along with worsening of existing cognitive decline (Gross et al., 2012). , Delirium may be the first or only sign that someone with dementia is unwell. Therefore, the timely investigation and management of the serious underlying causes of the delirium relies upon the rapid recognition and documentation of DSD. Assuming that the impairment is due to pre-existing dementia may result in diagnoses of potentially reversible conditions being missed. There is considerable uncertainty regarding the assessment of DSD (Richardson et al., 2016) and, partly as a consequence of this, it is often not recognised, particularly in acute medical admissions (Collins et al., 2010). In the absence of specific tools (Morandi et al., 2012), DSD is currently evaluated with instruments used for diagnosing delirium alone. This is problematic given that many of these tools rely on cognitive tests, which may be abnormal in both dementia and delirium (Meagher et al., 2010; Tieges et al., 2014).

Abnormal level of arousal and a patient's inability to focus, sustain and shift attention towards environmental stimuli are relatively specific to delirium (Brown et al., 2011b; Chester et al., 2012; Tieges et al., 2013). Importantly, in patients with abnormal arousal (above the level of coma), the inability to engage in cognitive testing or interview is considered severe inattention for the purposes of delirium diagnosis (European Delirium Association and American Delirium Society, 2014). Thus, arousal and attention are effectively part of the same spectrum, and both need to be assessed as part of delirium assessment (American Psychiatric Association, 2013). However, there is a lack of research on how best to combine arousal and attention tests in delirium assessment, particularly in DSD, as highlighted in a recent review (Morandi et al., 2016).

Arousal is not usually impaired in dementia, even in the advanced stages (Brown et al., 2011a). The Observational Scale of Level of Arousal (OSLA) has been shown to specifically identify delirium (Tieges et al., 2013) but has not previously been evaluated in the context of DSD. This measure is appealing as it is brief, observational, and does not require formal testing of cognition. 
Multiple tests of attention have been studied in the context of delirium diagnosis (Brown et al., 2011a; Meagher et al., 2010; Tieges et al., 2014; Tieges et al., 2015). However, there is a relative lack of research specifically addressing the role of attentional tests in recognizing delirium in patients with dementia. This is an important issue as attentional deficits may already be present in dementia, particularly when it is severe, and also because many of the tools test multiple cognitive domains affected in dementia alongside attention (Tieges et al., 2014). This suggests the need for a test of vigilant attention that could identify delirium, yet be simple enough to remain possible for those with dementia (Leonard et al., 2016).

This study aimed to evaluate existing tools to detect inattention (a vigilance task) and altered arousal (OSLA) in patients with delirium superimposed on dementia by comparing their individual and combined performances in four groups of older inpatients: no delirium, no dementia; delirium, no dementia; no delirium, dementia; delirium and dementia. We hypothesised that a combined arousal and attention testing procedure would more accurately detect DSD than the arousal or attention tests alone.

\section{METHODS}

\section{Subjects and design}

A convenient sample of patients over the age of 70 years admitted to five acute or rehabilitation hospitals in Italy, Ireland, Portugal and Switzerland were invited to take part in the study. The study protocol was approved by the ethics committee of each clinical centre. The following exclusion criteria were applied: presence of aphasia; history of major stroke; coma at the time of admission as defined by a Richmond Agitation and Sedation Scale $\leq-4$; poor vision or hearing. Informed consent was obtained from all participants, or their next of kin when the participants were not capable of giving informed consent because of delirium or other cognitive impairment.

\section{Dementia and delirium diagnosis}

Demographic data was collected and participants were then assessed for delirium and dementia by experienced delirium clinician-researchers (A Morandi, DM, WH, JC, GB). The diagnosis of delirium was made according to DSM-5 criteria by using a standardised procedure (Table 1) combining specific tests, information from nurses, carers and next of kin and review of the medical records. This information was supplemented by the assessor's judgement regarding subjective features and a final diagnosis made.

In non-delirious patients, a standardised MMSE (SMMSE) (Molloy and Standish, 1997) was completed in the local language. If the sMMSE score was $<28$, or if the participant had 
delirium, pre-existing dementia diagnosis was ascertained using the Informant Questionnaire on Cognitive Decline in the Elderly (IQCODE) in the local language with a cut-off of $\geq 3.5$ used to indicate likely dementia (Jorm et al., 1991). Following these assessments, participants were divided into the following 4 groups: no delirium, no dementia (control group); delirium, no dementia; no delirium, dementia; delirium, dementia (Figure 1).

\section{Attention test}

Attention was measured using a vigilance task, with participants signalling each time an " $A$ " was heard when the sequence of 10 consecutive letters "S-A-V-E-A-H-A-A-R-T" was read out, each letter 3 seconds apart. As per previous studies using this test, errors were counted when a patient failed to signal on the letter " $A$ " or when a patient signalled on any letter other than "A" (Ely et al., 2001). There was no published cut-point for the attention test, so the bestperforming cut-point was used.

\section{Level of arousal}

Arousal was measured using the Observational Scale of Level of Arousal (OSLA) (Tieges et al., 2013). The OSLA provides a total score ranging from 0 (awake and normal response) to 19 (unresponsive) composed of 5 items: eye opening, eye contact, posture, movement, and communication (Figure 2). Previously derived OSLA cut-point of 3/4 was used (Tieges et al., 2013).

\section{Statistical analysis}

Differences in characteristics of people with delirium, dementia, neither or both were assessed using $\mathrm{X}^{2}$ tests for proportions and nonparametric equality-of-medians tests for skewed continuous variables. Attention and level arousal scores were summed to derive a total score of 29 (S-A-V-E-A-H-A-A-R-T out of 10, OSLA out of 19), with cut-points for the combined scores derived from the point at which the highest proportion of participants were correctly classified. Diagnostic test accuracy was assessed using receiver operating characteristic (ROC) curves to yield sensitivity, specificity, positive and negative likelihood ratios and area under the ROC curve (AUROC), along with 95\% confidence intervals. All statistical procedures were carried out in Stata 13.1 (StataCorp, Texas). 


\section{RESULTS}

\section{Patient characteristics}

The sample included 114 people (Basel $n=15$; Coimbra $n=26$; Cremona $n=21$, Limerick $n=28$, Monza $n=24) ; 6$ patients were excluded for refusal of informed consent. Table 2 describes the characteristics of these participants. Mean age was 82 years (SD 7) and $54 \%(n=62)$ were female. Dementia alone was present in $25 \%(n=28)$, delirium alone in $18 \%(n=21)$, DSD in $27 \%(n=31)$, neither in $30 \%(n=34) .53 \%$ (31 of 59$)$ of those with dementia had delirium on admission.

Arousal-attention were assessed in $n=109$ (96\%). OSLA was scored in 114 participants $(100 \%)$ and the vigilance task in 109 (96\%). Of the five participants without scores for the vigilance task, three did not consent to testing and two were missing; these two had OSLA scores $=0$.

\section{Level of arousal}

The OSLA scores ranged from 0 to 14/19 (median=2, interquartile range 0,6 ). Using OSLA with the previously derived cut-off of 3/4 (Tieges et al., 2013), 83\% of participants with delirium were correctly identified (sensitivity 85\%, specificity 82\%, AUROC 0.92). Of those with dementia, delirium was correctly identified in $85 \%$ (sensitivity $74 \%$, specificity $96 \%$, AUROC 0.93).

\section{Attention test}

Errors ranged from 0 to $8 / 10$ (median=5, interquartile range 1,7 ). With a cut-off $3 / 4$, the attention task correctly classified $76 \%$ (sensitivity $90 \%$, specificity $64 \%$, AUROC 0.80 ) of participants with delirium. Of those with dementia, delirium was correctly identified in $79 \%$ (sensitivity $84 \%$, specificity $73 \%$, AUROC 0.79 ) at a cut-off of $6 / 7$.

\section{Combined test}

Combining scores (cut-off $9 / 10$ ) correctly classified $91 \%$ (sensitivity $84 \%$, specificity $97 \%$, AUROC 0.94) (Figure 3(a)). Even in those with underlying dementia $(n=59)$, the diagnostic accuracy for combining OSLA and attention tasks was very high, with $93 \%$ correctly classified (sensitivity 94\%, specificity 92\%, AUROC 0.98) (Figure 3(b)). 


\section{DISCUSSION}

The main finding in this study is that combining simple bedside assessments of arousal and attention sensitively and specifically identified delirium in patients with and without dementia. Moreover, a single score representing the sum of the two tests performed better than the two tests individually.

Despite previous studies exploring methods of measuring level of arousal (Chester et al., 2012; Han et al., 2015; Tieges et al., 2013), there was little consensus amongst delirium experts in a recent survey focusing on current clinical and research practice in DSD (Richardson et al., 2016). Our study provides further validation of the OSLA, a tool specifically designed for use in delirium (Tieges et al., 2013), and supports the view that measuring level of arousal using the OSLA has good specificity and sensitivity for delirium when used on admission to hospital, even in those with dementia.

Our findings support previous work which showed that vigilance, measured using a similar letter recognition test used in this study, distinguished patients with delirium from those with dementia alone, though there was some overlap in the scores (Leonard et al., 2016). A limitation of measuring attention using any tool is that it is not possible to assess all participants as a minimum level of arousal is required in order to complete the task. In this study, delirium experts were able to complete an assessment of vigilant attention in $96 \%$ of participants. An assessment of level of arousal using the OSLA is by its observational nature always possible in all participants; the function of the OSLA here is to provide additional gradation of arousal beyond simply stating that the patient was 'untestable'. We highlight the utility of combining the two tests in order to include a purely observational measure which supports previous work by Voyer et al concluding that 'one size does not fit all' and the use of a single cognitive test is not the best option in people with cognitive impairment (Voyer et al., 2016).

Other studies have examined alternative measures of vigilance and sustained attention and have reported comparable results (Brown et al., 2011a; Chester et al., 2012; Han et al., 2015; Tieges et al., 2015). Our work extends previous work by examining consecutive patients on acute admission to hospitals across four European countries demonstrating the reproducibility of the tools and their generalisability. Delirium assessments were performed by experts in the field according to the DSM-5 criteria using a standardised procedure. We did not exclude patients unable to communicate because of reduced arousal. Study limitations include the cross-sectional nature of assessment on admission, and so only prevalent delirium was examined. Although there was very little missing data, the sample size as a whole was relatively small, despite being larger than previous studies. This is particularly true when considering each site in turn. Incorporation bias is also difficult to avoid where there is a single 
assessor. This study did not evaluate the performance of this combined arousal-attention assessment in the context of different dementia severities and subtypes. Eliciting the best methods to measure both arousal and attention in these contexts should provide the focus of future work. The feasibility of such tools in untrained assessors requires evaluation along with validating its use in other settings e.g. intensive care or care homes. Interrater-reliability was not tested as the multicentre design of the study made this logistically challenging. However, the vast experience of those collecting the data along with the standardized approach for data collection ensured that the data was collected consistently between centres.

Our findings have direct clinical applicability. Currently, DSD is usually diagnosed through obtaining a collateral history, but often there is a lack of informant who can report an acute change from baseline. This may delay diagnosis or result in delirium being missed, resulting in worse outcomes (Kakuma et al., 2003). Therefore, combining simple and brief assessments of attention and arousal in order to sensitively and specifically identify DSD is appealing in this setting where time is limited and an informant is not always immediately available. Further work may explore other non-cognitive assessments of delirium including those that assess simple motor tasks. A recent study (Bellelli et al., 2011) has proposed a non-cognitive measure such as the assessment of motor fluctuations as a possible tool to distinguish DSD from advanced dementia. Individuals with DSD may have greater perturbation in motor agitation and retardation than those with dementia alone but better descriptions of motor disturbance are required.

This combined arousal-attention assessment to detect DSD was brief yet demonstrated high diagnostic accuracy even in dementia. Such an approach could have major clinical utility for diagnosing DSD. 


\section{CONFLICT OF INTEREST}

None

\section{DESCRIPTION OF AUTHORS' ROLES}

Study conception and design was done by all the authors except S. Richardson, who joined the team after this stage. Acquisition of data was done by G. Bellelli, D. Meagher, A. Morandi, W Hasemann and J. Cerejeira. Data analysis was done by A. Morandi, D. Davis and S. Richardson. Interpretation of results was done by all the authors. Manuscript was drafted by S. Richardson. Critical revision and final approval of the manuscript was done by all the authors.

\section{ACKOWLEDGMENTS}

David Meagher is supported by a research project grant from the Health Research Board in Ireland (HRA 2011/48). Sarah Richardson is funded by the Alzheimer's Society as a Clinical Training Fellow (239 (AS-CTF-14-001)). Daniel Davis is funded by the Wellcome Trust as an Intermediate Clinical Fellow (WT107467) 


\section{REFERENCES}

American Psychiatric Association (2013). Diagnostic and Statistical Manual of Mental Disorders, 5th edn, (DSM-5). Washington, DC: American Psychiatric Association.

Bellelli, G., et al. (2016). "Delirium Day": a nationwide point prevalence study of delirium in older hospitalized patients using an easy standardized diagnostic tool. BMC Medicine, 14, 112.

Bellelli, G., Speciale, S., Morghen, S., Torpilliesi, T., Turco, R. and Trabucchi, M. (2011). Are fluctuations in motor performance a diagnostic sign of delirium? Journal of the American Medical Directors Association, 12, 578-583.

Brown, L. J., Fordyce, C., Zaghdani, H., Starr, J. M. and MacLullich, A. M. (2011a). Detecting deficits of sustained visual attention in delirium. Journal of Neurology, Neurosurgery and Psychiatry, 82, 1334-1340.

Brown, L. J. E., et al. (2011b). Differential effects of delirium on fluid and crystallized cognitive abilities. Archives of Gerontology and Geriatrics, 52, 153-158.

Charlson, M. E., Pompei, P., Ales, K. L. and MacKenzie, C. R. (1987). A new method of classifying prognostic comorbidity in longitudinal studies: development and validation. Journal of Chronic Diseases, 40, 373-383.

Chester, J. G., Beth Harrington, M. and Rudolph, J. L. (2012). Serial administration of a modified Richmond Agitation and Sedation Scale for delirium screening. Journal of Hospital Medicine, 7, 450-453.

Collins, N., Blanchard, M. R., Tookman, A. and Sampson, E. L. (2010). Detection of delirium in the acute hospital. Age \& Ageing, 39, 131-135.

Ely, E. W., et al. (2001). Delirium in mechanically ventilated patients: validity and reliability of the confusion assessment method for the intensive care unit (CAM-ICU). Journal of the American Medical Association, 286, 2703-2710.

European Delirium Association and American Delirium Society (2014). The DSM-5 criteria, level of arousal and delirium diagnosis: inclusiveness is safer. BMC Medicine, 12, 141.

Fick, D. M., Agostini, J. V. and Inouye, S. K. (2002). Delirium superimposed on dementia: a systematic review. Journal of the American Geriatrics Society, 50, 1723-1732.

Gross, A. L., Jones, R. N., Habtemariam, D. A. and et al. (2012). Delirium and long-term cognitive trajectory among persons with dementia. Archives of Internal Medicine, 172, 13241331.

Han, J. H., et al. (2015). The Diagnostic Performance of the Richmond Agitation Sedation Scale for Detecting Delirium in Older Emergency Department Patients. Academic Emergency Medicine, 22, 878-882. 
Jorm, A. F., Scott, R., Cullen, J. S. and MacKinnon, A. J. (1991). Performance of the Informant Questionnaire on Cognitive Decline in the Elderly (IQCODE) as a screening test for dementia. Psychological Medicine, 21, 785-790.

Kakuma, R., et al. (2003). Delirium in older emergency department patients discharged home: effect on survival. Journal of the American Geriatrics Society, 51, 443-450.

Leonard, M., et al. (2016). Comparison of cognitive and neuropsychiatric profiles in hospitalised elderly medical patients with delirium, dementia and comorbid deliriumdementia. BMJ Open, 6, e009212.

Meagher, D. J., Leonard, M., Donnelly, S., Conroy, M., Saunders, J. and Trzepacz, P. T. (2010). A comparison of neuropsychiatric and cognitive profiles in delirium, dementia, comorbid delirium-dementia and cognitively intact controls. Journal of Neurology, Neurosurgery and Psychiatry, 81, 876-881.

Molloy, D. W. and Standish, T. I. (1997). A guide to the standardized Mini-Mental State Examination. International Psychogeriatrics, 9 Suppl 1, 87-94; discussion 143-150.

Morandi, A., et al. (2016). The Diagnosis of Delirium Superimposed on Dementia: An Emerging Challenge. Journal of the American Medical Directors Association. [E-pub ahead of print]

Morandi, A., et al. (2014). Delirium superimposed on dementia strongly predicts worse outcomes in older rehabilitation inpatients. Journal of the American Medical Directors Association, 15, 349-354.

Morandi, A., et al. (2012). Tools to Detect Delirium Superimposed on Dementia: A Systematic Review. Journal of the American Geriatrics Society, 60, 2005-2013.

Richardson, S., et al. (2016). Delirium superimposed on dementia: a survey of delirium specialists shows a lack of consensus in clinical practice and research studies. International Psychogeriatrics, 28, 853-861.

Tieges, Z., Brown, L. J. E. and MacLullich, A. M. J. (2014). Objective assessment of attention in delirium: a narrative review. International Journal of Geriatric Psychiatry, 29, 1185-1197.

Tieges, Z., McGrath, A., Hall, R. J. and Maclullich, A. M. (2013). Abnormal level of arousal as a predictor of delirium and inattention: an exploratory study. American Journal of Geriatric Psychiatry, 21, 1244-1253.

Tieges, Z., et al. (2015). Development of a smartphone application for the objective detection of attentional deficits in delirium. International Psychogeriatrics, 27, 1251-1262. Voyer, P., et al. (2016). Assessment of inattention in the context of delirium screening: one size does not fit all! International Psychogeriatrics, 28, 1293-1301. 
FIGURES/TABLES

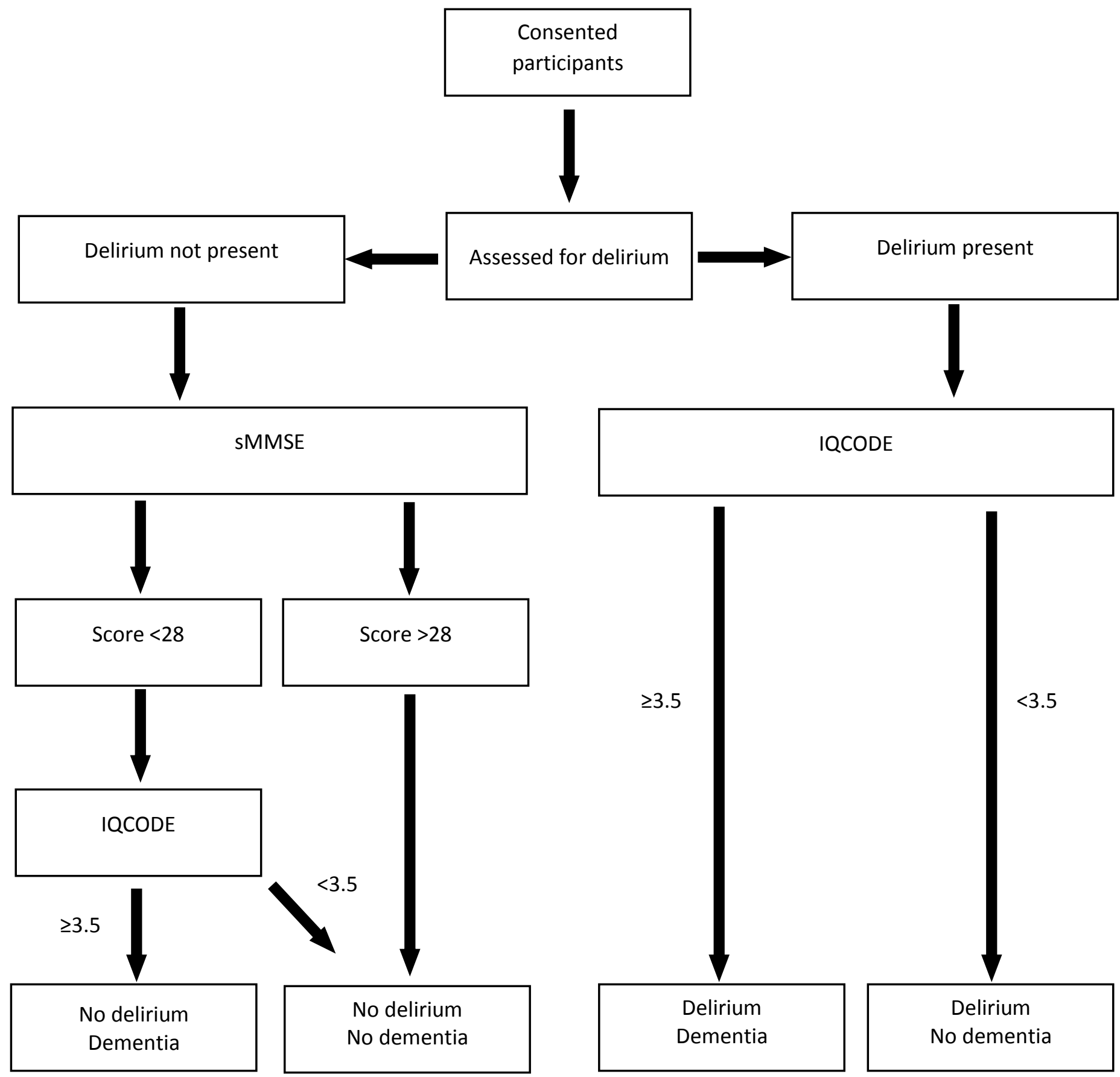

Figure 1: Flowchart demonstrating methods used to categorise participants 


\section{Eye opening}

0 Open on arrival and remain so, under patient's control, outlasts stimulus

1 Open on arrival but close if stimulus removed

1 Open to voice but then outlast stimulus

2 Open to voice but close if stimulus removed

3 Open to gentle physical stimulation (squeezing hand, gently shaking shoulder)

$4 \quad$ Open to pain only

$5 \quad$ No eye opening

\section{Eye contact}

$0 \quad$ Spontaneously makes and holds eye contact appropriately

1 Drowsy and makes eye contact to command but can't hold it for very long

1 Alert but eyes wandering, some appropriate eye contact

2 Alert but eyes wandering, little or no appropriate eye contact

2 Drowsy but makes brief eye contact

3 Eyes will / are open but no eye contact

Posture (NB take into account weakness due to stroke or neurological disease etc)

$0 \quad$ Sitting out in chair or up in bed, holding appropriate posture

1 Slumped in chair or bed but attempts to sit upright and sustain posture on request

2 Slumped in chair or bed and unable to sustain posture

3 Lying in bed and unable or no response to request to sustain posture

\section{Movement}

$0 \quad$ Moves spontaneously and purposefully with no restless or agitated movements

1 Occasional or mild restless or fidgety movements, no aggressive or vigorous movements

$1 \quad$ Reduced frequency of movement, mildly slowed up

2 Frequent restless or fidgety movements, no aggressive or vigorous movements

2 Moderately reduced frequency and speed of movement, interfering with assessment or self care

3 Aggressive or vigorous, recent pulling out of lines

4 Overtly combative, violent

$4 \quad$ Severely reduced frequency and speed of movement, few spontaneous movements

\section{Communication}

$0 \quad$ Orientated, alert and converses normally

1 Disorganised or disorientated speech but able to hold a conversation

2 Alert and inattentive, unable to focus on you long enough to hold a meaningful conversation, infrequent partial sentences

2 Drowsy, infrequent partial sentences in answer to questions

3 Alert and inattentive, unable to focus, one word answers

3 Drowsy, one word answers to questions

$4 \quad$ No verbal response

Score $(0-19)$

Figure 2: Observational Scale of Level of Arousal 


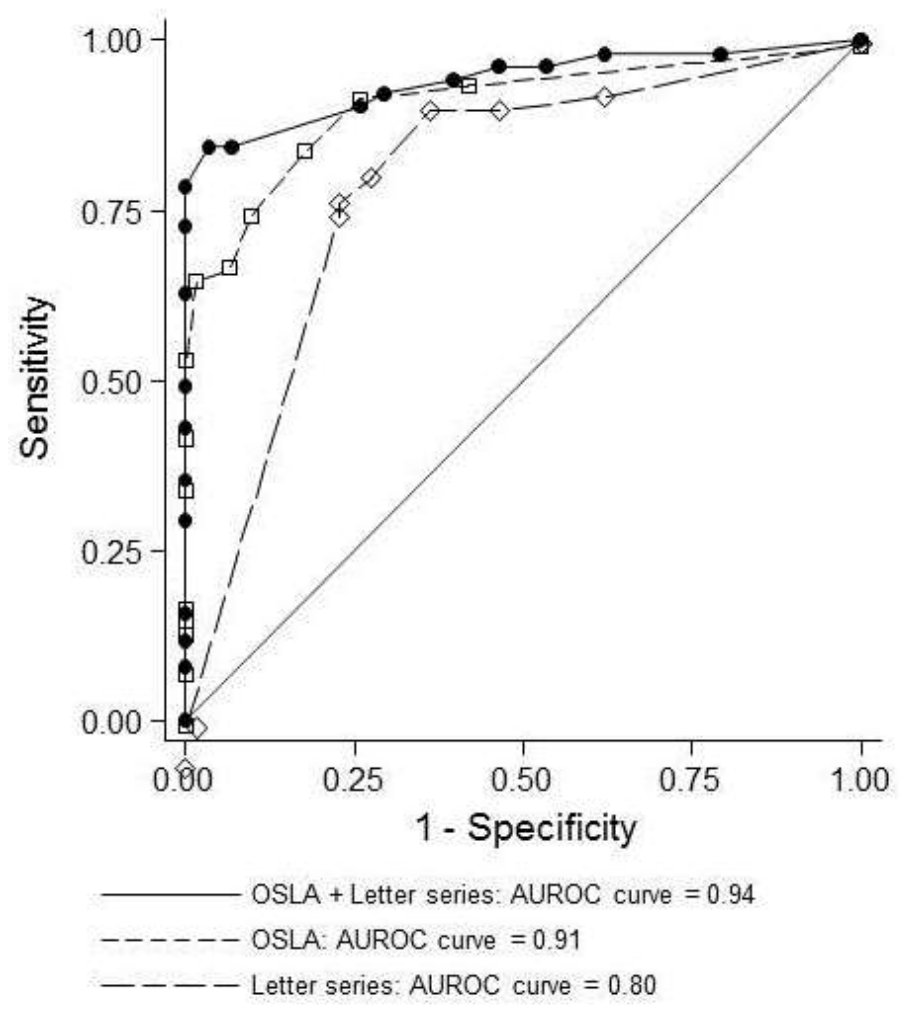

Figure 3(a): ROC curves for whole cohort $n=109$

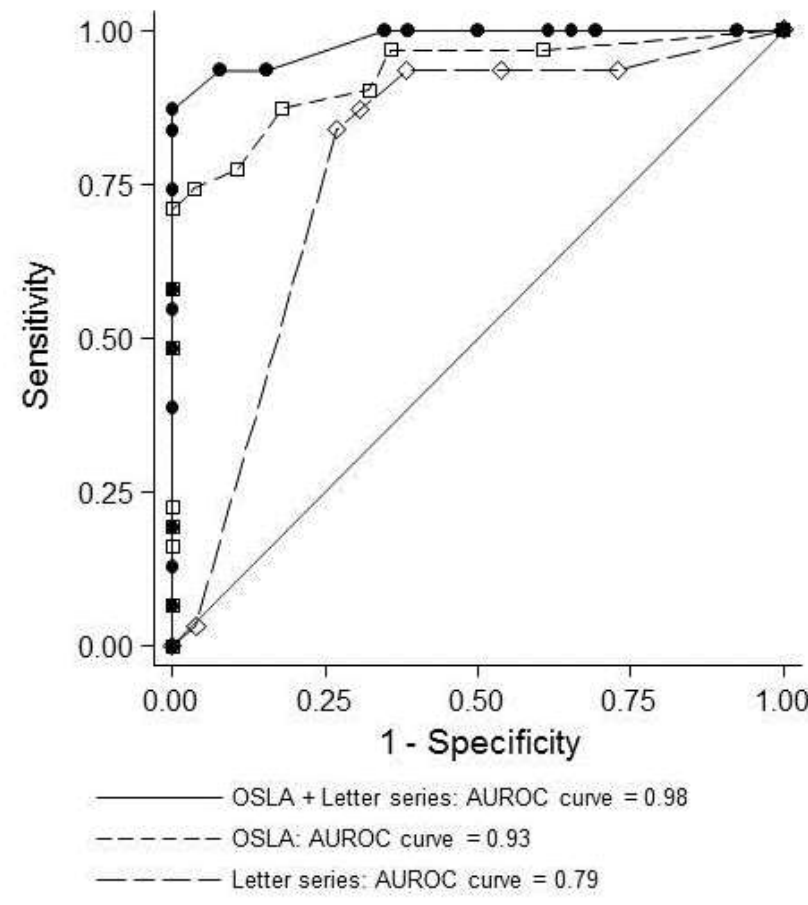

Figure 3(b): ROC curves for participants with dementia $n=57$ 


\begin{tabular}{|c|c|}
\hline DSM-5 criteria & $\begin{array}{l}\text { Test to be performed or information } \\
\text { needed }\end{array}$ \\
\hline \multirow{5}{*}{$\begin{array}{l}\text { A. Disturbance in attention (i.e., reduced } \\
\text { ability to direct, focus, sustain, and shift } \\
\text { attention) and awareness (reduced } \\
\text { orientation to the environment). }\end{array}$} & \begin{tabular}{l|l} 
Test & Cut-off \\
\end{tabular} \\
\hline & $\begin{array}{l}\text { Distractible; tending } \\
\text { to lose thread of } \\
\text { conversation; lacking } \\
\text { comprehension }\end{array}$ \\
\hline & $\begin{array}{l}\text { Orientation to time, } \\
\text { place, person }\end{array}$ \\
\hline & $\begin{array}{l}\text { Months of the year } \\
\text { backwards }\end{array}$ \\
\hline & Spatial span test \\
\hline $\begin{array}{l}\text { B. The disturbance develops over a short } \\
\text { period of time (usually hours to a few days), } \\
\text { represents a change from baseline attention } \\
\text { and awareness, and tends to fluctuate in } \\
\text { severity during the course of a day. }\end{array}$ & $\begin{array}{l}\text { Informant history from nursing staff, carers } \\
\text { and clinical notes }\end{array}$ \\
\hline $\begin{array}{l}\text { C. An additional disturbance in cognition } \\
\text { (e.g., memory deficit, disorientation, } \\
\text { language, visuospatial ability, or } \\
\text { perception). }\end{array}$ & $\begin{array}{l}\text { Impairment in any of the following domains: } \\
\text { MEMORY: inability to recall all of three items } \\
\text { at three minutes } \\
\text { ORIENTATION: disorientation to any of time, } \\
\text { place or person } \\
\text { LANGUAGE: impaired nerbal } \\
\text { communication in word naming or } \\
\text { comprehension } \\
\text { VISUOSPATIAL: impaired performance of } \\
\text { overlapping pentagons test or spatial } \\
\text { orientation questioning on } \\
\text { PERCEPTION: evidence of illusions or } \\
\text { hallucinations by collateral or direct } \\
\text { observation/questioning }\end{array}$ \\
\hline $\begin{array}{l}\text { D. The disturbances in criteria A and C are } \\
\text { not explained by another pre-existing, } \\
\text { established, or evolving neurocognitive } \\
\text { disorder and do not occur in the context of a } \\
\text { severely reduced level of arousal, such as } \\
\text { coma. }\end{array}$ & $\begin{array}{l}\text { Information from } \quad \text { history/chart/clinical } \\
\text { examination }\end{array}$ \\
\hline $\begin{array}{l}\text { E. There is evidence from the history, } \\
\text { physical examination, or laboratory findings } \\
\text { that the disturbance is a direct physiologic } \\
\text { consequence of another medical condition, } \\
\text { substance intoxication or withdrawal (i.e., } \\
\text { because of a drug of abuse or to a } \\
\text { medication), or exposure to a toxin or is } \\
\text { because of multiple aetiologies. }\end{array}$ & $\begin{array}{l}\text { Information } \\
\text { examination }\end{array}$ \\
\hline
\end{tabular}

Table 1: Operationalisation of the DSM-5 criteria for delirium 


\begin{tabular}{|l|l|l|l|l|l|}
\hline & $\begin{array}{l}\text { None } \\
\mathrm{n}=34\end{array}$ & $\begin{array}{l}\text { Dementia } \\
\mathrm{n}=28\end{array}$ & $\begin{array}{l}\text { Delirium } \\
\mathrm{n}=21\end{array}$ & $\begin{array}{l}\text { DSD } \\
\mathrm{n}=31\end{array}$ & $\mathrm{p}$ \\
\hline $\begin{array}{l}\text { Age } \\
(\text { Mean (SD)) }\end{array}$ & $81( \pm 6)$ & $82( \pm 7)$ & $84( \pm 6)$ & $84( \pm 7)$ & 0.03 \\
\hline $\begin{array}{l}\text { Female } \\
(\text { Number (\%)) }\end{array}$ & $19(56 \%)$ & $14(50 \%)$ & $11(52 \%)$ & $18(58 \%)$ & 0.9 \\
\hline $\begin{array}{l}\text { CCl } \\
(\text { Median (IQR)) }\end{array}$ & $2(1,3)$ & $3(2,4)$ & $3(2,4)$ & $3(2,5)$ & 0.3 \\
\hline $\begin{array}{l}\text { s-MMSE } \\
\text { (Median (IQR)) }\end{array}$ & $28(26,29)$ & $17(12,21)$ & - & - & $<0.01$ \\
\hline $\begin{array}{l}\text { IQCODE } \\
\text { (Median (IQR)) }\end{array}$ & $3(3,3.3)$ & $4.1(3.6,5)$ & $3(3,3.2)$ & $4.5(4,5)$ & $<0.01$ \\
\hline
\end{tabular}

Table 2: Patient characteristics 


\section{FIGURE/TABLE LEGENDS}

Figure 2: Flowchart demonstrating methods used to categorise participants

Participants were divided into four groups based on whether delirium was present or not according to DSM-5 criteria and then whether cognitive impairment was present or not, based upon the Standardised Mini-Mental State Examination (s-MMSE) (Molloy and Standish, 1997) or the Informant Questionnaire on Cognitive Decline in the Elderly (IQCODE) (Jorm et al., 1991)

Figure 2: Observational Scale of Level of Arousal

The OSLA (Tieges et al., 2013) was used to measure level of arousal. It provides a total score ranging from 0 (awake and normal response) to 19 (unresponsive) composed of 5 items: eye opening, eye contact, posture, movement, and communication.

Figure 3(a): ROC curves for whole cohort $n=109$

Figure 3(b): ROC curves for participants with dementia $n=57$

The combined attention-arousal testing procedure performed better than either tool individually when used to detect delirium. This was true in all participants studied (Figure 3(a)) and continued to perform well when examining just those participants with dementia (Figure 3(b)). [OSLA: Observational Scale of Level of Arousal (Tieges et al., 2013)]

Table 1: Operationalisation of the DSM-5 criteria for delirium The diagnosis of delirium was made according to DSM-5 criteria by using the standardised procedure described in the table. The final diagnosis of delirium was made based upon the information obtained from the specific tests, from nurses, carers and next of kin, review of the medical records and the assessor's judgement regarding subjective features.

Table 2: Patient characteristics

Abbreviations used in table 2:

CCl: Charlson co-morbidity index (Charlson et al., 1987)

DSD: Delirium Superimposed on Dementia

IQR: interquartile range

SD: standard deviation

s-MMSE: Standardised Mini-Mental State Examination (Molloy and Standish, 1997)

IQCODE: Informant Questionnaire on Cognitive Decline in the Elderly (Jorm et al., 1991) 